\title{
Los cuidados paliativos en atención primaria: un comentario sobre el manual del Instituto Nacional del Cáncer
}

\author{
Palliative care in primary care: a commentary on the National Cancer Institute manual
}

Javier O. Vilosio

\begin{abstract}
Resumen
Se comenta el recientemente publicado Manual de Cuidados Paliativos, enfatizando en los aspectos conceptuales que vinculan el abordaje de la especialidad con la actividad del equipo de atención primaria, con referencias al rol de los especialistas, y la responsabilidad ineludible de la totalidad de los equipos de salud.
\end{abstract}

\section{Abstract}

It discussed the recently published Handbook of Palliative Care, emphasizing the conceptual approach linking palliative care with the activity of the primary care team, with references to the role of specialists, and the inescapable responsibility of all health's teams.

Vilosio JO. Los Cuidados Paliativos en Atención Primaria: un comentario sobre el Manual del Instituto Nacional del Cáncer. Evid Act Pract Ambul. 2016;19(2):62-63. Abr-Jun.

Recientemente el Instituto Nacional del Cáncer (INC) de la República Argentina ha publicado la que esperamos sea la primera edición del Manual de cuidados paliativos para la atención primaria de la salud. En su introducción el documento hace referencia al problema de la fragmentación en la atención, y la necesidad de asumir la responsabilidad de la atención continua como un desafío de los equipos de atención primaria de la salud (APS). Y es que algunas actividades requeridas para esa continuidad resultan muy familiares a la concepción de estos equipos: la identificación de los pacientes y su entorno social y afectivo, el reconocimiento de sus necesidades y recursos disponibles para afrontar el tránsito por la terminalidad, su situación actual, la posible evolución de todas estas condiciones, y el diseño de un plan de acción, factible y efectivo.

En ese sentido el documento referencia la necesidad de diseñar circuitos de coordinación que eviten los "agujeros asistenciales" que generan abandono e inequidad. Los cuidados paliativos (CP) buscan mejorar la calidad de vida de las personas con enfermedades avanzadas de pronóstico letal, así como la de sus familias o entorno cercano y significativo, a través de la prevención y el control del sufrimiento con la identificación temprana, la evaluación y el tratamiento adecuados del dolor y otros malestares físicos, psico-sociales y existenciales. Desde la perspectiva propuesta, cuando la complejidad de las situaciones planteadas lo requiera, los equipos de atención primaria deberán contar con la posibilidad de recurrir a un equipo especializado en CP.

\section{El texto}

Luego de una introducción conceptual, el manual se organiza desarrollando algunos temas centrales: rol del profesional, plan de atención, comunicación, ética, síntomas, dolor, síntomas respiratorios, síntomas digestivos, delirium, síntomas psicológicos, familia, espiritualidad y final de vida. En su diseño se utilizan varias viñetas clínicas que sustentan el desarrollo de los principales conceptos, definiciones de "puntos clave" que resumen información sustancial, y dos anexos: sedación paliativa y fármacos para atender necesidades paliativas. El manual constituye una herramienta práctica para los aspectos instrumentales del control de síntomas pero fundamentalmente plantea cuestiones conceptuales relevantes para la comprensión del abordaje desde el enfoque de los cuidados paliativos e introduce una concepción estratégica de los cuidados que engarza con el concepto de APS. A los fines de este comentario nos detendremos someramente en estos aspectos.

\section{El papel del médico en atención primaria}

El documento se refiere a la APS como una estrategia (más allá de los niveles de atención), que responde a la necesidad de dar continuidad y atención paliativa a lo largo de todos los niveles del sistema de salud poniendo el eje en el médico de cabecera.

Los ámbitos de responsabilidad del médico en atención primaria se extienden en distintos campos. En la promoción y prevención (dada la particular posición para conocer y coordinar cuidados en aspectos psicológicos, sociales, familiares, paliativos y, llegado el caso, del duelo, tras la muerte del paciente), como en la detección precoz de riesgos, la continuidad del seguimiento (dada su posición privilegiada en cuanto al vínculo de confianza con el paciente y su entorno, en materia de cuestiones tales como la aceptación de la enfermedad, la información y comunicación, expectativas realistas sobre los tratamientos, complicaciones posibles y pasos a seguir), y en la coordinación entre niveles asistenciales, como canal de derivación, interconsulta y apoyo especializado desde el centro de atención primaria.

\section{Necesidades Paliativas}

El manual incluye la referencia a la situación de personas con enfermedades crónicas avanzadas y pronóstico de vida limitado con necesidades de atención paliativa, en las que es aconsejable iniciar gradualmente una orientación paliativa, combinada y no dicotómica (curativo vs. paliativo).

Aún menos difundido entre nosotros que el enfoque habitual de $\mathrm{CP}$, el abordaje desde la estrategia de Necesidades Paliativas (NECPAL) se fundamenta en la identificación de enfermos que requieren medidas paliativas de cualquier tipo, aún en servicios generales, no especializados.

El enfoque paliativo que se aplica en estos casos no contraindica ni limita las medidas de tratamiento específicas de la enfermedad, si tienen indicación precisa o pueden mejorar la calidad de vida.

Los seis pasos que se describen, para el desarrollo de este enfoque de atención paliativa son:

- Identificar necesidades multidimensionales.

- Practicar un modelo de atención impecable.

- Elaborar un plan terapéutico multidimensional.

- Identificar valores y preferencias del paciente: ética clínica y directivas anticipadas.

- Involucrar a la familia y al cuidador principal.

- Adaptar, organizar, llevar a cabo la gestión del caso, su seguimiento, la atención continuada y urgente, la coordinación de acciones integradas.

Esta atención puede ser implementada inicialmente y a largo plazo por cualquier equipo, en cualquier servicio de salud.

\section{Una breve discusión}

El manual se refiere explícitamente a los pacientes con cáncer, sin embargo es necesario recordar que el concepto de CP, con algunas peculiaridades, se aplica a las situaciones de terminalidad de causa oncológica y no oncológica. De particular rele-

‡ Sección Cuidados Paliativos del Servicio de Clínica Médica del Hospital Italiano de Buenos Aires. javier.vilosio@ @ospitalitaliano.org.ar 
vancia es la referencia que se hace sobre el NECPAL, que nos adelanta una respuesta posible e innovadora a un escenario epidemiológico que exige respuestas distintas a las habituales en el sistema de salud.

Por otra parte, si bien no hay duda que el equipo de atención primaria se encuentra en una posición privilegiada para el abordaje (por lo menos inicial) de las situaciones de final de vida, y para dar continuidad al cuidado de las personas en los sucesivos momentos de su ciclo vital, no es menos cierto que la responsabilidad de una actitud comprensiva y compasiva, de cuidado y acompañamiento, y de respeto a la autonomía de las personas y a sus necesidades y convicciones, incumbe a todos los profesionales y trabajadores de la salud. Sin excepciones y se debe enfatizar en ello.
En nuestra organización sanitaria un rasgo característico es la fragmentación. Y un problema de mayor relevancia, la inequidad, tanto en el acceso, como en la calidad de los servicios disponibles. El manual plantea la necesidad de dotar a los equipos de AP de los recursos requeridos (y agregamos: tanto materiales como organizacionales), incluyendo el desarrollo de los servicios especializados de $\mathrm{CP}$, que deberán dar apoyo a los equipos de AP. Su concreción dependerá de las condiciones institucionales y de financiamiento que lo hagan posible, es decir, de transformaciones pendientes.

Recibido el 01/06/2016 y aceptado el 15/06/2016

\section{Referencia:}

1. Jacob G. Manual de cuidados paliativos para la atención primaria de la salud. - 1a ed. - Ciudad Autónoma de Buenos Aires: Instituto Nacional del Cáncer, 2014. 276 p.: il. ; 13x19 cm. ISBN 978987-45494-3-3.Disponible en URL: http://www.msal.gov.ar/images/stories/bes/graficos/0000000592cnt-47-manual_paliativos_web.pdf

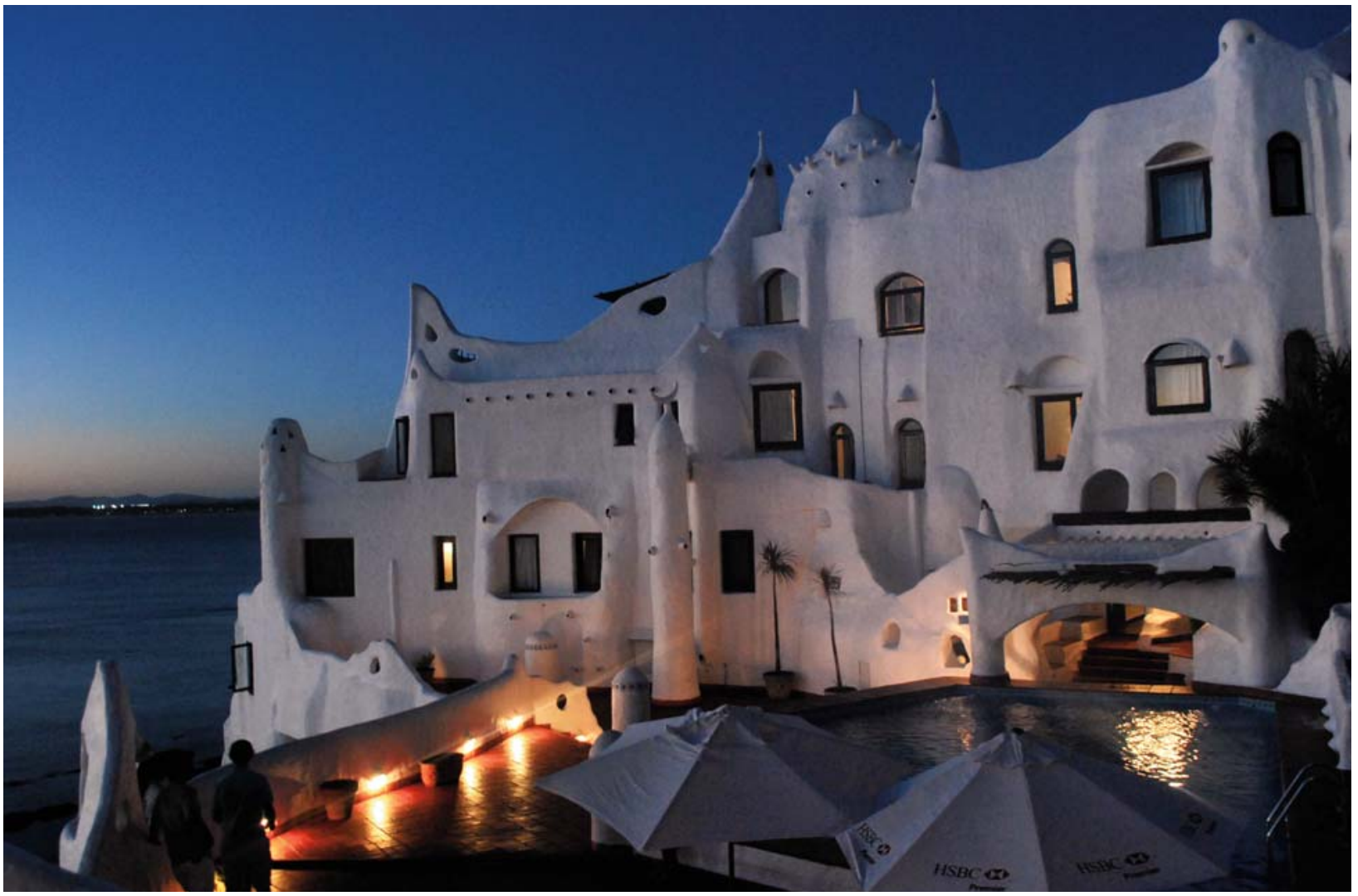

Cortesía: Silvina Spina 